\title{
Infecções virais e depressão
}

\author{
Viral infections and depression
}

\author{
Bárbara Perdigão Stumpf, Fábio Lopes Rocha, Anna Bárbara de Freitas Carneiro Proietti \\ e Grupo Interdisciplinar de Pesquisa em HTLV-1/2 (GIPH)
}

\section{Resumo}

Objetivo: A associação entre depressão e viroses é estudada há quase dois séculos, com resultados conflitantes. 0 objetivo deste trabalho é fazer uma análise crítica dos estudos existentes na literatura sobre essa relação. Métodos: A pesquisa bibliográfica utilizou as fontes eletrônicas de busca MEDLINE e LILACS (1966 a agosto 2005). As referências dos artigos foram utilizadas como fonte adicional de consulta. Resultados: Foram abordados os trabalhos que trataram da associação entre depressão e os vírus HIV, HCV, EBV, influenza, HSV, HBV, HAV, BDV e HTLV. A relação entre HIV e depressão mostrou-se bem documentada na literatura. Existem indícios de que a prevalência desse transtorno nos indivíduos infectados pelo HIV seja maior que a encontrada nos soronegativos. Além disso, estudos constataram que a depressão está associada a pior prognóstico da infecção. Quanto à associação entre HCV e depressão, os trabalhos sugeriram maior prevalência desse transtorno psiquiátrico nos portadores do HCV comparados à população geral. Não existem evidências científicas suficientes para dar suporte à relação entre os demais vírus e depressão. Conclusão: As associações mais bem fundamentadas foram aquelas entre depressão e os vírus HIV e HCV. A relação entre as demais viroses e depressão precisa ser mais bem estudada, e trabalhos com delineamento adequado se fazem necessários.

Palavras-chave: depressão, viroses, HIV, HCV, HTLV.

\begin{abstract}
Objective: The association between depression and viruses has been evaluated for almost two centuries now, with conflicting results. The objective of the present study is to perform a critical review of published studies in the literature about this relationship. Methods: Databases MEDLINE and LILACS were searched between 1966 and 2005 (until August). The references of the articles were used as additional source of data. Results: Studies about the link between depression and infection with viruses HIV, HCV, EBV, influenza, HSV, HBV, HAV, BDV and HTLV were analyzed. The association between HIV and depression was well documented in literature. There are evidences that the prevalence of this disorder in HIV-infected individuals is greater than that found in seronegatives. Moreover, studies concluded that depression is associated with a worse course of infection. Regarding the link between $\mathrm{HCV}$ and depression, studies suggested a greater prevalence of this psychiatric disorder in $\mathrm{HCV}$-infected people compared with general population. There is not enough scientific evidence to support the association between other viruses and depression. Conclusion: The best-studied relationships were those between depression and viruses HIV and HCV. Regarding the association of other viruses with depression, more research is needed.
\end{abstract}

Key words: depression, viruses, HIV, HCV, HTLV.

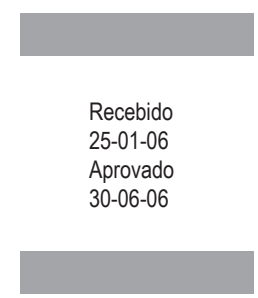

Instituto de Previdência dos Servidores do Estado de Minas Gerais (IPSEMG) (Stumpf BP)

Universidade de Brasília (UnB) (Rocha FL)

Universidade Federal de Minas Gerais (UFMG) (Proietti ABFC)

Correspondência para: Bárbara Perdigão Stumpf

Rua Desembargador Paula Mota, 170/301 - 31310-340 - Belo Horizonte-MG - Tel.: (31) 3498-4402/9979-5371

e-mail: bperdigao73@yahoo.com.br 


\section{Introdução}

É antiga a hipótese de que existe associação entre infecções virais e depressão (BMJ, 1976; White, 1999). Há vários relatos na literatura sobre a ocorrência de episódios depressivos após viroses (Kraepelin, 1890, apud Hotopf e Wessely, 1994; Caravati, 1944; Isaacs, 1948; Hendler e Leahy, 1978; White e Lewis, 1987). O objetivo deste artigo é fazer uma revisão crítica dos estudos existentes sobre a relação entre infecções virais e depressão. Serão abordados os trabalhos que tratam da associação entre depressão e os seguintes vírus: vírus da imunodeficiência humana (HIV), vírus da hepatite C (HCV), vírus Epstein-Barr (EBV), vírus influenza, vírus herpes simples (HSV), vírus da hepatite $B(H B V)$, vírus da hepatite $A(H A V)$, vírus da doença de Borna (BDV) e vírus linfotrópico humano de células $T$ (HTLV).

\section{Métodos}

Foi realizada uma pesquisa eletrônica na base de dados MEDLINE e na Literatura Latino-Americana e do Caribe em Ciências da Saúde (LILACS), considerando artigos em línguas portuguesa, inglesa e espanhola de 1966 a agosto de 2005. Na MEDLINE, os termos de busca empregados inicialmente foram depression e viruses. Posteriormente, a expressão depression foi associada a cada um dos seguintes vírus: influenza, EBV, HSV, HAV, HBV, HCV, BDV, HIV e HTLV. Na base de dados LILACS, os descritores utilizados inicialmente foram depressão e viroses. O termo depressão foi em seguida associado a cada um dos vírus citados anteriormente. A partir dos resultados obtidos foram selecionados os artigos de interesse para o tema em questão. Para complementar a pesquisa bibliográfica, as referências dos artigos selecionados foram pesquisadas para buscar possíveis artigos não encontrados através da busca eletrônica inicial.

\section{Resultados}

\section{Vírus da imunodeficiência humana}

Entre os transtornos psiquiátricos mais comumente observados em indivíduos HIV-positivos, a depressão é o mais prevalente (Malbergier e Schöffel, 2001; Chandra et al., 2005). A depressão maior em indivíduos infectados pelo HIV parece estar associada a fatores como estigma da doença, efeitos diretos do vírus e infecções oportunistas no sistema nervoso central (SNC), além do desencadeamento de episódio depressivo em populações vulneráveis como usuários de drogas injetáveis (UDIs) e homossexuais (Malbergier e Schöffel, 2001; Chandra et al., 2005).

\section{Prevalência de depressão em indivíduos infectados pelo HIV}

Os estudos sobre a prevalência de depressão em indivíduos HIV-positivos apresentaram resultados muito variados $(0 \%$ a $45 \%$ ). Isso se deve a alguns fatores como população estudada (homossexuais masculinos, mulheres e UDIs), instrumento de avaliação utilizado (entrevista diagnóstica padronizada e escalas de auto-avaliação), local de realização da pesquisa (comunidade e serviços médicos) e estágio da doença (Penzak et al., 2000; Fulk et al., 2004; Chandra et al., 2005). Além disso, o diagnóstico de depressão em indivíduos infectados pelo HIV pode ser dificultado devido: à tendência dos profissionais de saúde em considerar a depressão reação normal ao diagnóstico da infecção; à presença de sintomas somáticos que complicam o diagnóstico diferencial; ao receio de alguns pacientes em expressarem seus sentimentos e aos efeitos colaterais psiquiátricos de alguns anti-retrovirais (Penzak et al., 2000; Fulk et al., 2004; Chandra et al., 2005).

Dois trabalhos avaliaram a prevalência de depressão em indivíduos HIV-positivos de ambos os sexos (Maj et al., 1994; McDaniel et al., 1995) (Tabela 1). O primeiro, de Maj et al. (1994), foi um estudo multicêntrico e controlado que incluiu 955 indivíduos (602 HIV-positivos e 353 controles) de cinco países (Brasil, Tailândia, Zaire, Alemanha e Quênia). A prevalência média de depressão foi $7 \%$ (variação de 0\% a 21\%) no grupo infectado e $2 \%$ (variação de $0 \%$ a $8 \%$ ) no grupo controle (Ciesla e Roberts, 2001). A diferença entre os grupos não foi significativa. 0 segundo, um estudo não-controlado, mostrou prevalência de depressão de $29 \%$ nos homens infectados pelo HIV e $35 \%$ nas mulheres soropositivas (McDaniel et al., 1995). Ambos os trabalhos, entretanto, incluíram indivíduos de populações variadas (heterossexuais, homossexuais e usuários de drogas, entre outros) e que se encontravam em diferentes estágios da infecção pelo vírus.

Com relação aos estudos controlados que focalizaram a prevalência de depressão na população masculina infectada pelo HIV (Atkinson et al., 1988; Williams et al., 1991; Chuang et al., 1992; Rosenberger et al., 1993; Perkins et al., 1994; Lyketsos et al., 1996a; Rabkin et al., 1997a; Fukunishi et al., 1997; Kelly et al., 1998), nenhum deles mostrou prevalência significativamente maior desse transtorno nos indivíduos soropositivos comparados aos controles, embora na maioria dos trabalhos observe-se tendência a maior prevalência no grupo infectado (Tabela 2). No único estudo que encontrou $0 \%$ de prevalência de depressão em ambos os grupos, os pacientes HIV-positivos apresentaram pontuações significativamente maiores no item depressão de uma escala de auto-avaliação (Fukunishi et al., 1997). Dos nove trabalhos analisados, seis incluíram somente homossexuais ou bissexuais (Williams et al., 1991; Rosenberger

Tabela 1. Estudos sobre a prevalência de depressão em indivíduos infectados pelo HIV

\begin{tabular}{|c|c|c|c|c|}
\hline Autor & $n$ & $\begin{array}{l}\text { Instrumento } \\
\text { de avaliação }\end{array}$ & $\begin{array}{c}\text { Depressão } \\
\text { atual (\%) }\end{array}$ & $p$ \\
\hline Maj et al.,1994a & $\begin{array}{l}602 \text { HIV+ } \\
353 \text { HIV- }\end{array}$ & $\begin{array}{l}\text { CIDI }^{\mathrm{b}} \\
\text { MADRS }^{\mathrm{c}} \\
\text { BPRS }^{\mathrm{d}}\end{array}$ & $\begin{array}{c}0-21(7,1)^{e} \\
0-7,8[2]^{e}\end{array}$ & $\mathrm{NS}^{t}$ \\
\hline $\begin{array}{l}\text { McDaniel et al., } \\
1995\end{array}$ & $37 \mathrm{HIV}+$ & $\begin{array}{l}\text { SCIDg }^{g} \\
\text { BDI }^{\mathrm{h}} \\
\text { HAM-Di }\end{array}$ & $\begin{array}{l}29 \text { (homens) } \\
35 \text { (mulheres) }\end{array}$ & $N A^{j}$ \\
\hline
\end{tabular}

aEstudo multicêntrico; ' $\mathrm{CIDI}$ : Composite International Diagnostic Interview; 'MADRS: Montgomery-Asberg Depression Rating Scale; 'BPRS: Brief Psychiatry Rating Scale; ' $v a l o r$ médio estimado por Ciesla e Roberts (2001); 'NS: não-significativo; '9SCID: Structured Clinical Interview for DSM-III-R; ' $B D I$ : Beck Depression Inventory; 'HAM-D: Hamilton Rating Scale for Depression; 'NA: não se aplica. 
Tabela 2. Estudos controlados sobre a prevalência de depressão em homens infectados pelo HIV

\begin{tabular}{|c|c|c|c|c|}
\hline Autor & $n$ & Instrumento de avaliação & Depressão atual (\%) & $p$ \\
\hline Atkinson et al., 1988 & $\begin{array}{l}43 \text { HIV+ } \\
33 \text { HIV- }\end{array}$ & $\mathrm{DIS}^{\mathrm{a}}$ & $\begin{array}{c}7-17(11)^{b} \\
0-9(3)^{b}\end{array}$ & $\mathrm{NS}^{\mathrm{c}}$ \\
\hline Williams et al., 1991d & $\begin{array}{l}123 \text { HIV+ } \\
84 \text { HIV- }\end{array}$ & $\begin{array}{l}\text { SCIDe } \\
\text { SCL-90f }\end{array}$ & $\begin{array}{c}4 \\
3,5\end{array}$ & $N S^{c}$ \\
\hline Chuang et al., 1992 & $\begin{array}{l}144 \mathrm{HIV+} \\
29 \mathrm{HIV}-\end{array}$ & POMSg $^{9}$ & $\begin{array}{c}5-10(6,9)^{b} \\
6,6\end{array}$ & $\mathrm{NS}^{\mathrm{c}}$ \\
\hline Rosenberger et al., $1993^{\mathrm{d}}$ & $\begin{array}{l}166 \text { HIV+ } \\
31 \text { HIV- }\end{array}$ & SCIDe & $\begin{array}{c}9-11(10)^{b} \\
6\end{array}$ & $\mathrm{NS}^{\mathrm{c}}$ \\
\hline Perkins et al., 1994 & $\begin{array}{l}98 \text { HIV+ } \\
71 \text { HIV- }\end{array}$ & $\begin{array}{l}\text { SCIDe } \\
\text { HAM-D }\end{array}$ & $\begin{array}{l}8 \\
3\end{array}$ & $N S^{c}$ \\
\hline Lyketsos et al., 1996 d,i & $\begin{array}{l}750 \mathrm{HIV+} \\
2.412 \mathrm{HIV}-\end{array}$ & CES-Dj & $\begin{array}{l}9-12(10)^{b} \\
9-12(10)^{b}\end{array}$ & $N S^{c}$ \\
\hline Rabkin et al., 1997d & $\begin{array}{l}183 \mathrm{HIV+} \\
84 \mathrm{HIV}-\end{array}$ & $\begin{array}{c}\mathrm{SCID}^{\mathrm{e}} \\
\mathrm{HAM}^{\mathrm{H}} \mathrm{D}^{\mathrm{h}} \\
\mathrm{BDI}^{\mathrm{I}} \\
\mathrm{BSI}^{\mathrm{m}}\end{array}$ & $\begin{array}{c}7,6 \\
8\end{array}$ & $N S^{c}$ \\
\hline Fukunishi et al., 1997 & $\begin{array}{l}50 \text { HIV+ } \\
47 \text { HIV- }\end{array}$ & $\begin{array}{l}\mathrm{SCID}^{\mathrm{e}} \\
\mathrm{POMS}^{9}\end{array}$ & $\begin{array}{l}0 \\
0\end{array}$ & $N S^{c}$ \\
\hline Kelly et al., $1998^{d}$ & $\begin{array}{l}164 \text { HIV+ } \\
65 \text { HIV- }\end{array}$ & DIS $^{a}$ & $\begin{array}{l}(18)^{b} \\
(9)^{b}\end{array}$ & $N S^{c}$ \\
\hline
\end{tabular}

aDIS: Diagnostic Interview Schedule; 'valor médio estimado por Ciesla e Roberts (2001); 'NS: não-significativo; 'estudo que incluiu somente homossexuais ou bissexuais; ' $S C I D$ : Structured Clinical Interview for DSM-III-R; ' $S C L-90$ : Symptom Check List 90; 9POMS: Profile of Mood States; hHAM-D: Hamilton Rating Scale for Depression; 'estudo multicêntrico; 'CES-D: Center for Epidemiologic Studies Depression Scale; 'BDI: Beck Depression Inventory; 'mBSI: Brief Symptom Inventory.

et al., 1993; Perkins et al., 1994; Lyketsos et al., 1996a; Rabkin et al., 1997a; Kelly et al., 1998).

Quanto aos nove trabalhos sobre a prevalência de depressão em mulheres HIV-positivas (James, 1988; Brown e Rundell, 1990; Pergami et al., 1993; Lovisi e Morgado, 1996; Goggin et al., 1998; Ickovics et al., 2001; Morrison et al., 2002; Evans et al., 2002; Tostes et al., 2004), apenas três são controlados (Pergami et al., 1993; Morrison et al., 2002; Evans et al., 2002) (Tabela 3). Desses, somente um mostrou prevalência de depressão significativamente maior nas mulheres infectadas pelo HIV comparadas às soronegativas (Morrison et al., 2002). Nos estudos não-controlados que não investigaram populações especiais de mulheres portadoras do HIV (Lovisi e Morgado,
1996; Goggin et al., 1998; Ickovics et al., 2001; Tostes et al., 2004), como grávidas e militares, a maior prevalência (acima de $40 \%$ ) foi observada em trabalho que incluiu pacientes com história de abuso de substâncias no último ano (Ickovics et al., 2001). Um levantamento em mulheres grávidas portadoras do HIV relatou prevalência de depressão de 7\% (James, 1988). Nesse trabalho as pacientes foram avaliadas assim que souberam do diagnóstico da infecção, e nenhum instrumento de avaliação foi utilizado. No único estudo que pesquisou a prevalência de depressão em mulheres militares soropositivas, a taxa encontrada foi de 0\% (Brown e Rundell, 1990).

Nos estudos de revisão que compararam a prevalência de depressão em indivíduos soropositivos de ambos

Tabela 3. Estudos sobre a prevalência de depressão em mulheres infectadas pelo HIV

\begin{tabular}{|c|c|c|c|c|}
\hline Autor & $n$ & Instrumento de avaliação & Depressão atual (\%) & $p$ \\
\hline James, $1988^{\mathrm{a}}$ & $15 \mathrm{HIV}+$ & Nenhum & 7 & $N A^{b}$ \\
\hline Brown e Rundell, $1990^{\circ}$ & $20 \mathrm{HIV+}$ & HAM-D ${ }^{d}$ & 0 & $N A^{b}$ \\
\hline Pergami et al., 1993 & $\begin{array}{l}57 \mathrm{HIV+} \\
23 \mathrm{HIV}-\end{array}$ & $\mathrm{ZSDS}^{\mathrm{e}}$ & $\begin{array}{l}45 \\
56\end{array}$ & $N S^{f}$ \\
\hline Lovisi e Morgado, 1996 & $44 \mathrm{HIV+}$ & $\left.\mathrm{CID}\right|^{g}$ & 27 & $N A^{b}$ \\
\hline Goggin et al., 1998 & $54 \mathrm{HIV+}$ & $\begin{array}{l}\text { SCID }^{\mathrm{h}} \\
\text { HAM-D }^{\mathrm{d}} \\
\text { BSI }^{i}\end{array}$ & 1,9 & $N A^{b}$ \\
\hline Ickovics et al., 2001j & 765 HIV+ & CES-D' & 42 & $N A^{b}$ \\
\hline Morrison et al., 2002 & $\begin{array}{l}93 \text { HIV+ } \\
62 \text { HIV- }\end{array}$ & $\begin{array}{l}\text { SCID } \\
\text { HAM-D }\end{array}$ & $\begin{array}{c}19 \\
5\end{array}$ & SIGN ${ }^{m}$ \\
\hline Evans et al., 2002 & $\begin{array}{l}63 \text { HIV+ } \\
30 \text { HIV- }\end{array}$ & $\begin{array}{l}\mathrm{SCID}^{\mathrm{h}} \\
\text { HAM-D }\end{array}$ & $\begin{array}{l}16 \\
10\end{array}$ & $N S^{f}$ \\
\hline Tostes et al., 2004 & $76 \mathrm{HIV+}$ & HADS $^{n}$ & 30,3 & $N A^{b}$ \\
\hline
\end{tabular}

aEstudo que incluiu somente mulheres grávidas; ' ${ }^{\mathrm{N} A}$ : não se aplica; 'estudo que incluiu somente mulheres militares; ${ }^{\text {dHAM}} \mathrm{D}$ : Hamilton Rating Scale for Depression;

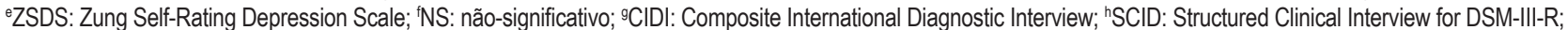
'BSI: Brief Symptom Inventory; 'estudo multicêntrico; 'CES-D: Center for Epidemiologic Studies Depression Scale; 'mSIGN: significativo (<0,05); " $H A D S$ : Hospital Anxiety and Depression Scale. 
os sexos, a prevalência nas mulheres pareceu ser superior àquela encontrada nos homens (Penzak et al., 2000; Chandra et al., 2005). Entretanto, a prevalência de depressão é mais elevada em mulheres do que em homens na população geral, independentemente do status sorológico para o HIV (Penzak et al., 2000).

Com relação à prevalência de depressão em UDIs infectados pelo HIV, existem três estudos controlados (Lipsitz et al., 1994; Malbergier e Andrade, 1999; Turrina et al., 2001) (Tabela 4). Desses, dois abordaram usuários de heroína injetável (Lipsitz et al., 1994; Turrina et al., 2001). O primeiro deles constatou que a prevalência de depressão nesse grupo (11\%-27\%) foi superior àquela encontrada na população geral, independentemente da sorologia desses sintomas nos últimos estágios da infecção pelo vírus (Lyketsos et al., 1996b). Nesse trabalho, entretanto, não foram utilizados instrumentos padronizados de diagnóstico em psiquiatria, não sendo possível fazer estimativas de prevalência.

Como a maior parte dos trabalhos sobre a prevalência de depressão em portadores do HIV incluiu número pequeno de indivíduos, estudos de metanálise são úteis na detecção de associações não observadas em trabalhos isolados. Uma dessas metanálises (Ciesla e Roberts, 2001) incluiu dez estudos, num total de 2.596 participantes (Atkinson et al., 1988; Williams et al., 1991; Chuang et al., 1992; Rosenberger et al., 1993; Lipsitz et al., 1994; Maj et al., 1994; Perkins et al., 1994; Fukunishi et al., 1997; Rabkin et al., 1997a; Kelly et al., 1998).

Tabela 4. Estudos controlados sobre a prevalência de depressão em usuários de drogas injetáveis infectados pelo HIV

\begin{tabular}{|c|c|c|c|c|}
\hline Autor & $n$ & Instrumento de avaliação & Depressão atual (\%) & $p$ \\
\hline Lipsitz et al., 1988 & $\begin{array}{l}124 \text { HIV+ } \\
99 \text { HIV- }\end{array}$ & $\begin{array}{c}\mathrm{SCID}^{\mathrm{a}} \\
\mathrm{SCL}-90 \mathrm{R}^{\mathrm{b}}\end{array}$ & $\begin{array}{c}18 \text { (mulheres) } \\
22 \text { (homens) } \\
11 \text { (homens) } \\
27 \text { (mulheres) }\end{array}$ & $\mathrm{NS}^{\mathrm{c}}$ \\
\hline Malbergier e Andrade, 1999 & $\begin{array}{l}30 \text { HIV+ } \\
30 \text { HIV- }\end{array}$ & $\mathrm{CIDI}^{\mathrm{d}}$ & $\begin{array}{l}27 \\
20\end{array}$ & $\mathrm{NS}^{\mathrm{c}}$ \\
\hline Turrina et al., 2001 & $\begin{array}{l}127 \mathrm{HIV+} \\
51 \mathrm{HIV}-\end{array}$ & $\begin{array}{l}\mathrm{HADS}^{\mathrm{e}} \\
\mathrm{CIDI}^{d}\end{array}$ & $\begin{array}{l}36 \\
16\end{array}$ & SIGN ${ }^{f}$ \\
\hline
\end{tabular}

aSCID: Structured Clinical Interview for DSM-III-R; ' ${ }^{S C L}-90 R$ : Symptom Check List 90 Revised; cNS: não-significativo; ${ }^{d}$ CIDI: Composite International Diagnostic Interview; eHADS: Hospital Anxiety and Depression Scale; 'SIGN: significativo $(<0,05)$

para o HIV (Lipsitz et al., 1994). No entanto, o segundo mostrou uma prevalência de depressão significativamente maior nos indivíduos infectados pelo HIV comparados aos soronegativos (36\% versus $16 \% ; p<0,05)$ (Turrina et al., 2001). A maioria dos pacientes soropositivos (56\%), porém, preenchia critérios para pré-síndrome de imunodeficiência adquirida (pré-AIDS) ou AIDS clínica.

Em usuários de cocaína injetável, Malbergier e Andrade (1999), em nosso meio, concluíram que a sorologia positiva para o HIV não foi fator associado à maior prevalência de depressão. Em termos comparativos, a prevalência de depressão nos UDIs infectados pelo HIV (18\%-36\%) pareceu ser mais elevada que nos homossexuais masculinos soropositivos (0\%-18\%).

Alguns trabalhos compararam a prevalência de depressão nos diferentes estágios da infecção pelo HIV (Atkinson et al., 1988; Chuang et al., 1992; Rosenberger et al., 1993; Maj et al., 1994, Rabkin et al., 1997a; Rabkin et al., 1997b; Kelly et al., 1998). Estudos transversais não encontraram prevalências de depressão significativamente maiores nos pacientes HIV-positivos sintomáticos comparados aos assintomáticos (Atkinson et al., 1988; Chuang et al., 1992; Rosenberger et al., 1993; Maj et al., 1994; Rabkin et al., 1997a; Kelly et al., 1998). Em uma coorte que acompanhou homossexuais masculinos soropositivos durante quatro anos não foi observado aumento significativo na prevalência de depressão ao longo do período, apesar da progressão da doença (Rabkin et al., 1997b). Com relação à presença de sintomas depressivos, mas não necessariamente depressão, um estudo prospectivo que acompanhou homossexuais masculinos HIV-positivos durante sete anos mostrou aumento significativo
Entre os trabalhos selecionados, seis incluíram apenas homossexuais masculinos no grupo soro positivo (Atkinson et al., 1988; Williams et al., 1991; Rosenberger et al., 1993; Perkins et al., 1994; Rabkin et al., 1997a; Kelly et al., 1998); um incluiu somente UDIs (Lipsitz et al., 1994) e os restantes incluíram indivíduos de várias populações (Chuang et al., 1992; Maj et al., 1994; Fukunishi et al., 1997). Apesar de nenhum estudo individualmente ter demonstrado maior prevalência de depressão em indivíduos HIV-positivos comparados a soronegativos, a conclusão da metanálise foi que indivíduos infectados pelo HIV apresentaram prevalência de depressão duas vezes maior que a encontrada nos HIV-negativos (Ciesla e Roberts, 2001). Em relação à orientação sexual, não foi constatado maior risco de depressão em homossexuais masculinos e bissexuais HIV-positivos comparados aos heterossexuais soropositivos. Também não houve diferença significativa nas taxas de depressão nos grupos com doença avançada comparados aos portadores assintomáticos do vírus. Apesar da maior prevalência de depressão nos soropositivos, apenas um em cada dez indivíduos apresentou episódio depressivo maior atual. Tais dados sugeriram que a infecção não estaria diretamente associada à depressão. Entretanto, devido às diferentes populações incluídas nos estudos, não se pode concluir definitivamente sobre a questão.

\section{Repercussões da depressão na progressão da infecção pelo HIV}

Alguns autores estudaram a relação entre depressão e evolução da infecção pelo HIV (Leserman et al., 1999; Ickovics et al., 2001; Evans et al., 2002; Bouhnik et al., 2005) (Tabela 5). 
Tabela 5. Estudos sobre as repercussões da depressão na evolução da infecção pelo HIV

\begin{tabular}{|c|c|c|}
\hline Autor & População do estudo & Achados \\
\hline Leserman et al., 1999 & 82 homossexuais masculinos HIV+ & $\begin{array}{l}\text { Associação entre progressão da infecção e presença } \\
\text { de sintomas depressivos, estresse e falta de suporte } \\
\text { social }\end{array}$ \\
\hline Ickovics et al., 2001 & 765 mulheres HIV+ & $\begin{array}{l}\text { Mortalidade duas vezes maior nas mulheres com } \\
\text { sintomas depressivos crônicos }\end{array}$ \\
\hline Evans et al., 2002 & $\begin{array}{l}63 \text { mulheres HIV+ } \\
30 \text { mulheres HIV- }\end{array}$ & $\begin{array}{l}\text { Associação entre depressão e } \downarrow \text { atividade das } \\
\text { células NK, } \uparrow \text { linfócitos T CD8 e } \uparrow \text { da carga viral nas } \\
\text { soropositivas }\end{array}$ \\
\hline Bounnik et al., 2005a & $\begin{array}{l}175 \text { homens UDIsb } \mathrm{HIV+} \\
68 \text { mulheres UDIs }{ }^{\mathrm{H}} \mathrm{HIV+}\end{array}$ & $\begin{array}{l}\text { Associação entre progressão da infecção e presença } \\
\text { de sintomas depressivos }\end{array}$ \\
\hline
\end{tabular}

${ }^{a}$ Estudo em que todos os pacientes estavam em uso de Highly Active Antiretroviral Therapy (HAART); bUDIs: usuários de drogas injetáveis.

Apesar de os estudos serem baseados em populações variadas, em todos foi mostrado que a depressão está associada a pior prognóstico da infecção.

\section{Vírus da hepatite $\mathrm{C}$}

A associação entre HCV e depressão começou a ser estudada na década de 1990, logo após a disponibilização de testes sorológicos para o vírus. Duas linhas de evidências dão suporte a essa relação. Uma delas é o fato de pacientes portadores de transtornos psiquiátricos apresentarem maior prevalência de infecção pelo HCV que a população geral. A outra é a maior prevalência de transtornos psiquiátricos em indivíduos infectados pelo HCV, sendo a depressão o distúrbio mais freqüente e clinicamente importante (Zdilar et al., 2000).

Assim como na infecção pelo HIV, os estudos sobre a prevalência de depressão em indivíduos HCV-positivos apresentaram resultados variados. Os motivos de tal varia- ção foram os mesmos relatados previamente nos trabalhos com HIV, ressaltando-se os efeitos colaterais psiquiátricos da terapia antiviral à base de interferon (Zdilar et al., 2000; Wessely e Pariante, 2002).

Dos sete trabalhos sobre a prevalência de depressão em indivíduos infectados pelo HCV (Taruschio et al., 1996; Lee et al., 1997; Pariante et al., 1999; Sherman et al., 1999; Dwight et al., 2000; Goulding et al., 2001; Koskinas et al., 2002), quatro são controlados (Pariante et al., 1999; Sherman et al., 1999; Goulding et al., 2001; Koskinas et al., 2002) (Tabela 6). $O$ único estudo controlado que utilizou um instrumento diagnóstico padronizado mostrou maior prevalência de depressão em indivíduos HCV-positivos comparados a portadores do HBV (Pariante et al., 1999). Em outros dois trabalhos com grupo controle, a prevalência de depressão nos pacientes infectados pelo HCV foi superior à encontrada nos soronegativos (Sherman et al., 1999; Goulding et al., 2001). O estudo que apresentou a maior prevalência, entretanto, incluiu UDls (Goulding et al., 2001). No estudo controlado mais recente, contudo, não foi constatada

Tabela 6. Estudos sobre a prevalência de depressão em indivíduos infectados pelo HCV

\begin{tabular}{|c|c|c|c|c|}
\hline Autor & $n$ & Instrumento de avaliação & Depressão atual (\%) & $p$ \\
\hline Taruschio et al., 1996 & $30 \mathrm{HCV}+$ & $\begin{array}{c}\mathrm{BDl}^{\mathrm{a}} \\
\mathrm{MADRS}^{\mathrm{b}} \\
\mathrm{BPRS}^{\mathrm{c}} \\
\mathrm{CGl}^{\mathrm{d}}\end{array}$ & 10 & $N A^{e}$ \\
\hline Lee et al., 1997 & $359 \mathrm{HCV}+$ & Nenhum & 24 & $N A^{e}$ \\
\hline Pariante et al., 1999 & $\begin{array}{l}39 \mathrm{HCV}+ \\
18 \mathrm{HBV}+\end{array}$ & SCID & $\mathrm{HCV}>\mathrm{HBV}$ & $N D^{g}$ \\
\hline Sherman et al., 1999 & $\begin{array}{l}96 \mathrm{HCV}+ \\
398 \mathrm{HCV}-\end{array}$ & $\begin{array}{l}\text { CES-D } \\
\text { SCL-90R }\end{array}$ & $\mathrm{HCV}>$ controles & NDg \\
\hline Dwight et al., 2000 & $50 \mathrm{HCV}+$ & $\begin{array}{l}\text { DIS } \\
\mathrm{BDI}^{\mathrm{a}}\end{array}$ & 28 & $N A^{e}$ \\
\hline Goulding et al., 2001 & $\begin{array}{l}77 \mathrm{HCV}+ \\
25 \mathrm{HCV}-\end{array}$ & HADS' & $\begin{array}{c}45 \\
4\end{array}$ & SIGNm \\
\hline Koskinas et al., 2002 & $\begin{array}{l}38 \mathrm{HCV}+ \\
58 \mathrm{HCV}- \\
36 \mathrm{HBV}+\end{array}$ & $\mathrm{ZSDS}^{n}$ & $\mathrm{HCV}=\mathrm{HBV}=$ controles & NS ${ }^{\circ}$ \\
\hline
\end{tabular}

aBDI: Beck Depression Inventory; bMADRS: Montgomery-Asberg Depression Rating Scale; cBPRS: Brief Psychiatry Rating Scale; 'CGI: Clinical Global Impression; eNA: não se aplica; 'SCID: Structured Clinical Interview for DSM-III-R; ' NDD: não disponível no trabalho original; 'CES-D: Center for Epidemiologic Studies Depression Scale; 'SCL-90R: Symptom Check List 90 Revised; iDIS: Diagnostic Interview Schedule; 'HADS: Hospital Anxiety and Depression Scale; ' ${ }^{2}$ IGN: significativo (<0,05); nZSDS: Zung Self-Rating Depression Scale; `NS: não-significativo. 
diferença significativa na prevalência de depressão em indivíduos infectados por HCV ou HBV comparados a controles sem infecção (Koskinas et al., 2002).

Com relação aos estudos não-controlados (Taruschio et al., 1996; Lee et al., 1997; Dwight et al., 2000), a menor prevalência foi relatada em trabalho em que igual número de pacientes preencheu critérios diagnósticos para transtorno misto ansioso e depressivo (Taruschio et al., 1996). Nos levantamentos de pacientes procedentes de serviços terciários, as prevalências variaram entre 24\% e 28\% (Lee et al., 1997; Dwight et al., 2000). Um desses trabalhos também mostrou que os pacientes HCVpositivos deprimidos apresentavam número significativamente maior de sintomas somáticos comparados aos não-deprimidos (Dwight et al., 2000).

Alguns autores estudaram a prevalência de depressão em populações especiais de portadores do HCV, como usuários de drogas, pacientes terminais e militares (Singh et al., 1997; Johnson et al., 1998; Singh et al., 1999; Muir e Provenzale, 2002; El-Serag et al., 2002; Lehman e Cheung, 2002; Fireman et al., 2005) (Tabela 7). Um estudo controlado em usuários de drogas infectados pelo HCV no qual os indivíduos desconheciam o status sorológico para o vírus constatou prevalências elevadas de depressão, tanto no grupo soropositivo quanto nos controles (57,5\% versus $48,2 \% ; p=0,09)$ (Johnson et al., 1998).

Um estudo de pacientes com doença hepática terminal mostrou maior prevalência de depressão nos infectados pelo HCV comparados aos demais (Singh et al., 1997). Foi observado também que os portadores do HCV apresentavam mais sintomas somáticos e dor que os outros pacientes. Os mesmos autores acompanharam um grupo de pacientes submetidos a transplante de fígado e constataram maior prevalência de depressão naqueles com hepatite $C$ recorrente comparados aos soronegativos (Singh et al., 1999). Quanto aos quatro trabalhos sobre a prevalência de depressão em militares infectados pelo HCV (Muir e Provenzale, 2002; El-Serag et al., 2002; Lehman e Cheung, 2002; Fireman et al., 2005), somente um é controlado (El-Serag et al., 2002). Esse estudo mostrou maior prevalência de depressão em militares HCV-positivos comparados a soronegativos $(49,5 \%$ versus $39,1 \% ; p<0,0001)$. Dos três trabalhos não-controlados (Muir e Provenzale, 2002; Lehman e Cheung, 2002; Fireman et al., 2005), apenas um encontrou taxa inferior a 40\% (Muir e Provenzale, 2002). Tais estudos, no entanto, não utilizaram instrumentos padronizados de diagnóstico e incluíram predominantemente indivíduos do sexo masculino com história de abuso de substância.

Em resumo, os estudos existentes sobre a prevalência de depressão em indivíduos infectados pelo HCV sugerem que essa seja superior à encontrada na população geral. Esses dados reforçam a hipótese de que existe associação entre HCV e depressão.

\section{Vírus Epstein-Barr}

A associação entre EBV e depressão foi descrita na literatura há mais de 50 anos. No estudo pioneiro, um grupo de pacientes com mononucleose infecciosa (MI), mais comumente causada pelo EBV, foi acompanhado por mais de três meses, e a presença de sintomas depressivos foi observada naqueles com infecção prolongada (Isaacs, 1948). Outros trabalhos relataram episódios depressivos após MI (Prick e De Sonaville, 1953, apud White, 1999; Du Bois et al., 1984) incluindo casos graves com ideação suicida (Hendler e Leahy, 1978) e sintomas psicóticos (White e Lewis, 1987). Um estudo mostrou aumento significativo da prevalência de depressão em mulheres que tiveram MI no ano anterior, mas não em homens (Cadie et al., 1976). Outro trabalho em estudantes canadenses encontrou maior prevalência de depressão naqueles que tiveram Ml comparados aos que tiveram outras infecções de vias aéreas superiores (IVAS) (Lambore et al., 1991).

Os estudos prospectivos sobre o tema apresentaram resultados conflitantes (Bruce-Jones et al., 1994; White et al., 1998; Katon et al., 1999). No único estudo não-controlado, a prevalência de depressão em pacientes com Ml (4,9\%) foi similar àquela encontrada na população geral (Katon et al., 1999). Em outro trabalho, pacientes portadores de MI, amigdalite nãocausada por EBV ou IVAS foram acompanhados durante seis meses, sendo constatado que as infecções virais em geral foram importantes desencadeadoras de depressão na fase aguda da infecção (Bruce-Jones et al., 1994). Entretanto, outro estudo encontrou prevalência de depressão significativamente maior nos indivíduos com Ml comparados aos portadores de amigdalite não-causada por EBV ou IVAS (White et al., 1998). A duração média dos episódios depressivos, contudo, foi de apenas três semanas (White et al., 1998).

Tabela 7. Estudos sobre a prevalência de depressão em populações especiais de indivíduos infectados pelo HCV

\begin{tabular}{|c|c|c|c|c|}
\hline Autor & $n$ & Instrumento de avaliação & Depressão atual (\%) & $p$ \\
\hline Johnson et al., 1998 ${ }^{a}$ & $\begin{array}{l}163 \mathrm{HCV}+ \\
147 \mathrm{HCV}-\end{array}$ & CES-D ${ }^{b}$ & $\begin{array}{l}57,5 \\
48,2\end{array}$ & $\mathrm{NS}^{\mathrm{c}}$ \\
\hline Singh et al., $1997^{d}$ & $\begin{array}{l}42 \mathrm{HCV}+ \\
40 \mathrm{HCV}-\end{array}$ & $\left.\mathrm{BD}\right|^{\mathrm{e}}$ & $\mathrm{HCV}>$ controles & SIGN \\
\hline Singh et al., 1999d & $\begin{array}{l}34 \mathrm{HCV}+ \\
25 \mathrm{HCV}-\end{array}$ & $\mathrm{BDI}^{\mathrm{e}}$ & $\mathrm{HCV}>$ controles & SIGN \\
\hline Muir e Provenzale, $2002^{g}$ & $100 \mathrm{HCV}+$ & CES-D ${ }^{b}$ & 12 & $N A^{h}$ \\
\hline El-Serag et al., $2002^{\mathrm{g}}$ & $\begin{array}{l}22.341 \mathrm{HCV}+ \\
43.267 \mathrm{HCV}-\end{array}$ & Nenhum & $\begin{array}{l}49,5 \\
39,1\end{array}$ & SIGN \\
\hline Lehman e Cheung, $2002^{g}$ & $120 \mathrm{HCV}+$ & $\left.\mathrm{BD}\right|^{\mathrm{e}}$ & 44,2 & $N A^{h}$ \\
\hline Fireman et al., $2005^{9}$ & $393 \mathrm{HCV}+$ & $\mathrm{BDl}$ & 81,2 & $N A^{h}$ \\
\hline
\end{tabular}

${ }^{a}$ Estudo que incluiu somente usuários de drogas; ' $\mathrm{CES}-\mathrm{D}$ : Center for Epidemiologic Studies Depression Scale; 'NS: não-significativo; destudo que incluiu somente pacientes terminais; ${ }^{\mathrm{B} D I}$ : Beck Depression Inventory; ${ }^{\mathrm{S}} \mathrm{I} \mathrm{GN}$ : significativo $(<0,05)$; ${ }^{9}$ estudo que incluiu somente militares; ${ }^{\mathrm{h} N A}$ : não se aplica. 
Assim como nos estudos longitudinais, os trabalhos que investigaram a relação entre depressão e evidência sorológica de infecção pelo EBV apresentaram resultados controversos (Amsterdam et al., 1986; Cooke et al., 1991).

\section{Vírus influenza}

A associação entre vírus influenza e depressão foi descrita há mais de cem anos. Kraepelin (1890, apud Hotopf e Wessely, 1994) relatou 11 casos de depressão pós-influenza no início da epidemia de 1890. Trabalhos posteriores ressaltaram que os episódios depressivos pós-influenza eram leves e transitórios na maioria dos casos (Espagnol, 1895, apud Menninger, 1921). Um estudo da década de 1950 também relatou casos de depressão após essa virose. Em nenhum deles, porém, a influenza foi confirmada por testes sorológicos (Pillay, 1959, apud Sinanan e Hillary, 1981). Apesar da fraca evidência científica, um editorial do British Medical Journal (1971) afirmou que surtos de influenza poderiam ser seguidos por depressão intratável.

Um levantamento inglês posterior a esse editorial, entretanto, não encontrou maior número de internações psiquiátricas devido à depressão após epidemias de influenza (Flewett, 1976). Adicionalmente, em trabalho que comparou a prevalência de anticorpos antiinfluenza em pacientes deprimidos e em portadores de outros transtornos psiquiátricos, não foi observada diferença significativa entre os grupos (Sinanan e Hillary, 1981). Uma pesquisa realizada durante epidemia de influenza num hospital geral também não demonstrou maior prevalência de sintomas depressivos nos pacientes com essa virose específica comparados aos que apresentavam outras infecções de vias aéreas superiores (Hashimoto et al., 1987). Contudo, um estudo em adolescentes que apresentavam títulos elevados de anticorpos antiinfluenza mostrou prevalência de sintomas depressivos significativamente maior naqueles que tiveram infecção sintomática nos últimos seis meses comparados ao grupo assintomático (Meijer et al., 1988). As possíveis explicações para esse achado são a conhecida tendência de títulos elevados de anticorpos antiinfluenza persistirem por mais de uma estação e a possibilidade de alguns indivíduos assintomáticos terem infecção subclínica.

Em síntese, existem poucos estudos sobre a associação entre vírus influenza e depressão, e os resultados encontrados foram conflitantes.

\section{Vírus herpes simples}

A associação entre HSV e depressão foi descrita na literatura nas décadas de 1960 e 1970, não existindo estudos recentes sobre o tema. Aúnica metodologia utilizada nos trabalhos foi a pesquisa de evidências de infecção pelo HSV em pacientes deprimidos.

O primeiro estudo, realizado em 1969, mostrou uma prevalência de anticorpos anti-HSV significativamente maior nos pacientes com depressão psicótica comparados a portadores de outros transtornos psiquiátricos e a controles (Rimon e Halonen, 1969). Um outro trabalho comparou a prevalência de anticorpos anti-HSV em deprimidos psicóticos, controles e indivíduos com infecção aguda pelo HSV. Os pacientes deprimidos psicóticos apresentaram prevalência significativamente maior de anticorpos anti-HSV comparados aos controles. Já os indivíduos com infecção viral aguda apresentaram maior prevalência de anticorpos anti-HSV que os pacientes deprimidos psicóticos, porém a prevalência decaiu seis a oito semanas após a infecção (Cappel et al., 1978).

Em outro trabalho, entretanto, não foi encontrada diferença significativa na prevalência de anticorpos anti-HSV em pacientes deprimidos comparados a portadores de outros transtornos psiquiátricos e controles (Pokorny et al., 1973). Adicionalmente, outro estudo mostrou maior prevalência de anticorpos anti-HSV em pacientes psiquiátricos em geral comparados a controles (Halonen et al., 1974). Outro trabalho também constatou prevalências mais elevadas de anticorpos anti-HSV tanto em pacientes deprimidos psicóticos quanto em portadores de demência comparados a controles (Lycke et al., 1974).

Em resumo, existem poucos estudos sobre a associação entre HSV e depressão, e os resultados encontrados foram controversos.

\section{Vírus da hepatite $B$}

A associação entre HBV e depressão foi pouco estudada até o momento. Conforme mencionado no tópico Vírus da hepatite $C$, em estudo que comparou a prevalência de depressão em indivíduos infectados por HBV ou HCV foi encontrada maior prevalência nos HCV-positivos (Pariante et al., 1999). Em outro trabalho, a prevalência de depressão foi estudada em indivíduos com hepatite viral (B ou C) e em controles saudáveis, não sendo constatada diferença significativa entre os grupos (Koskinas et al., 2002).

\section{Vírus da hepatite $\mathrm{A}$}

A associação entre HAV e depressão foi descrita na literatura, porém os trabalhos existentes são antigos, datando 0 último de 1974 (Caravati, 1944; Sherlock e Walshe, 1946; Martini e Strohmeyer, 1974). Além de escassos, os estudos apresentaram resultados conflitantes. Caravati (1944) relatou casos de soldados que apresentavam síndrome pós-hepatite caracterizada por instabilidade emocional, fadiga, desconforto no quadrante superior direito do abdome, intolerância à gordura e desnutrição após hepatite A. Quanto à instabilidade emocional especificamente, $83 \%$ dos indivíduos apresentavam evidências de depressão e ansiedade. Achados similares foram encontrados por outros autores (Martini e Strohmeyer, 1974). Entretanto, um estudo não constatou a presença de sintomas psiquiátricos em soldados com síndrome pós-hepatite (Sherlock e Walshe, 1946). Uma deficiência metodológica comum a todos os trabalhos é a ausência de confirmação sorológica da infecção pelo HAV.

Dessa forma, pode-se concluir que a associação entre HAV e depressão foi pouco estudada até o momento.

\section{Vírus da doença de Borna}

O BDV é um vírus neurotrópico pertencente à família Bornaviridae (Bode e Ludwig, 2003). O agente causa um tipo raro de meningoencefalite em cavalos e ovelhas e é endêmico há mais de 150 anos em certas regiões da Alemanha e da Suíça. Após inoculação experimental do BDV em animais de laboratório, foi observada uma síndrome neurocomportamental específica, semelhante ao transtorno afetivo bipolar em humanos (Amsterdam et al., 1985). Embora não existam relatos de doenças humanas causadas pelo BDV (Kim et al., 1999), a associação entre esse vírus e depressão é estudada há aproximadamente 20 anos. 
Os trabalhos existentes se basearam na pesquisa de evidências de infecção pelo BDV em pacientes deprimidos, e os resultados encontrados foram conflitantes (Carbone, 2001; Bode e Ludwig, 2003).

\section{Vírus linfotrópico humano de células T}

A associação entre HTLV e depressão foi pouco estudada até o momento. Em 2000, Proietti relatou a presença de sintomas depressivos em indivíduos infectados pelo HTLV-1, acompanhados em estudo de coorte aberta prevalente de doadores de sangue soropositivos em um hemocentro de Minas Gerais. Resultados preliminares de um estudo recente em indivíduos da coorte citada sugeriram maior prevalência de depressão nos portadores do HTLV-1 comparados a doadores de sangue soronegativos (45,5\% versus 18,8\%; $p=0,0543$ ) (Stumpf et al., 2005). Tais achados foram confirmados posteriormente (comunicação pessoal).

\section{Discussão}

Os resultados dos trabalhos publicados na literatura mostraram que as associações mais bem fundamentadas foram aquelas entre depressão e os vírus HIV e HCV. A relação entre as demais viroses estudadas e depressão tem mínima evidência científica.

Quatro principais estratégias metodológicas foram utilizadas no estudo dessa associação. A primeira delas consistiu em estudos descritivos, ou seja, relatos de depressão em pacientes com determinada virose (por exemplo: Pillay, 1959, apud Sinanan e Hillary, 1981; Hendler e Leahy, 1978). Essa metodologia apresenta várias limitações que comprometem a interpretação dos resultados. Entre elas destacam-se: (a) a maioria dos trabalhos inclui um número reduzido de indivíduos e tende a selecionar os casos mais graves, nos quais qualquer associação detectada será exagerada; (b) todos os participantes sabem do diagnóstico da infecção viral; (c) poucos estudos utilizam instrumentos padronizados de diagnóstico em psiquiatria e (d) a ausência de um grupo controle.

A segunda estratégia empregada, estudos transversais controlados (por exemplo: Maj et al., 1994; Rabkin et al., 1997a; Pariante et al., 1999), apresenta vantagens em relação à primeira. A principal delas é a presença de um grupo de comparação. Entretanto nesses trabalhos não é possível estabelecer relação de causa e efeito, isto é, não se sabe se a depressão é anterior ou posterior à infecção virótica.

A terceira estratégia utilizada foi baseada em tentativas de demonstração de evidências de infecção viral em pacientes deprimidos (por exemplo: Cappel et al., 1978; Amsterdam et al., 1986; Bode e Ludwig, 2003). Os resultados desses estudos devem ser analisados com cautela, uma vez que tanto a supressão da ativação linfocitária pode tornar a pessoa deprimida mais suscetível à infecção viral quanto a ativação imune decorrente da depressão pode levar a um aumento inespecífico dos títulos de anticorpos, 0 que poderia ser interpretado como evidência de que a virose seria a causa da depressão (Hotopf e Wessely, 1994).

A quarta estratégia consistiu em estudos prospectivos de pacientes com infecções virais específicas (por exemplo: Rabkin et al., 1997b; White et al., 1998; Singh et al., 1999). Tais trabalhos, apesar da superioridade em relação aos anteriores, também devem ser analisados com cuidado. Sabe-se que os indivíduos deprimidos tendem a procurar os serviços médicos com maior freqüência que os não-deprimidos (Katon e Schulberg, 1992). Isso pode fazer com que eles constituam a maior parte das coortes de pacientes com infecções virais provenientes dos serviços primários de atenção à saúde. Além disso, pacientes acompanhados em coortes conhecem seu diagnóstico, sendo impossível separar o impacto psicológico do mesmo dos efeitos diretos do vírus.

Um complicador no estudo da relação entre infecções virais e depressão é o fato de que tanto a depressão quanto os eventos de vida estressantes também provocam alterações no sistema imune. Os trabalhos sobre os efeitos da depressão no sistema imune evidenciaram decréscimos em todas as medidas de função linfocitária (Herbert e Cohen, 1993; Hotopf e Wessely, 1994). Além disso, a atividade das células destruidoras naturais (células NK) também se encontra significativamente reduzida (Weisse, 1992; Herbert e Cohen, 1993; Hotopf e Wessely, 1994). A depressão também está associada à elevação do número de leucócitos circulantes, principalmente neutrófilos e monócitos (Herbert e Cohen, 1993). Uma possível explicação para tais achados é o fato de a depressão estar relacionada à ativação do eixo hipotálamo-hipófise-adrenal e ao sistema nervoso simpático, o que resultaria em níveis séricos elevados de cortisol e catecolaminas (Weisse, 1992; Herbert e Cohen, 1993; Hotopf e Wessely, 1994). Como as células do sistema imunológico possuem receptores para esses hormônios, suspeita-se que elas exerçam papel na modulação do mesmo. Entretanto, não existem estudos demonstrando ligação entre depressão, cortisol e imunidade. Uma outra hipótese envolve a associação entre depressão e comportamentos específicos que influenciam a resposta imune. Pessoas deprimidas podem apresentar alterações da imunidade devido à insônia, ao sedentarismo, à diminuição do apetite e ao uso de álcool e de outras drogas (Weisse, 1992; Herbert e Cohen, 1993; Hotopf e Wessely, 1994). Os eventos de vida estressantes também parecem estar relacionados com a diminuição da função imune (Weisse, 1992; Hotopf e Wessely, 1994).

De maneira geral, para se estudar a associação entre vírus e depressão, dois tipos de trabalhos são recomendados. 0 primeiro, mais limitado, são os estudos transversais controlados em que o diagnóstico da virose e a presença de sintomas depressivos são avaliados simultaneamente. Na tentativa de diminuir os fatores de confusão, recomenda-se que sejam utilizadas amostras randomizadas da população geral ou de populações específicas, como doadores de sangue. A melhor abordagem são os estudos realizados com indivíduos participantes de coortes de casos incidentes de determinada infecção viral.

\section{Conclusão}

As associações mais bem fundamentadas foram aquelas entre depressão e os vírus HIV e HCV. A relação entre as demais viroses e depressão tem mínima evidência científica, e estudos com delineamento adequado se fazem necessários.

\section{Agradecimentos}

À Fundação Centro de Hematologia e Hemoterapia de Minas Gerais (Hemominas), à Coordenação de Aperfeiçoamento de Pessoal de Nível Superior (CAPES), à Fundação de Amparo à Pesquisa do Estado de Minas Gerais (FAPEMIG) e ao Conselho Nacional de Desenvolvimento Científico e Tecnológico (CNPq), pelo apoio. 


\section{Referências}

Amsterdam JD, Winokur A, Dyson W, Herzog S, Gonzalez F, Rott R, et al. Borna disease virus: a possible etiologic factor in human affective disorders? Arch Gen Psychiatry, 42: 1093-6, 1985.

Amsterdam JD, Henle W, Winokur A, Wolkowitz OM, Pickar S, Paul SM. Serum antibodies to Epstein-Barr virus in patients with major depressive disorder. Am J Psychiatry, 143: 1593-6, 1986.

Atkinson JH, Grant I, Kennedy CJ, Richman DD, Spector SA, McCutchan A. Prevalence of psychiatric disorders among men infected with human immunodeficiency virus. Arch Gen Psychiatry, 45: 859-64, 1988.

Bode L, Ludwig H. Borna disease virus infection, a human mentalhealth risk. Clin Microbiol Rev, 16: 534-45, 2003.

Bouhnik A, Préau M, Vincent E, Carrieri MP, Gallais H, Lepeu G, et al. Depression and clinical progression in HIV-infected drug users treated with highly active antiretroviral therapy. Antiviral Ther, 10: 53-61, 2005.

Brown GR, Rundell JR. Prospective study of psychiatric morbidity in HIV-seropositive women without AIDS. Gen Hosp Psychiatry, 12: 30-5, 1990.

Bruce-Jones WDA, White PD, Thomas JM, Clare AW. The effect of social adversity on the fatigue syndrome, psychiatric disorders and physical recovery, following glandular fever. Psychol Med, 24: $651-9,1994$

Cadie M, Nye FJ, Storey P. Anxiety and depression after infectious mononucleosis. Br J Psychiatry, 128: 559-61, 1976.

Cappel R, Gregoire F, Thiry L, Sprecher S. Antibody and cell-mediated immunity to herpes simplex virus in psychotic depression. J Clin Psychiatry, 39: 266-8, 1978.

Caravati CM. Posthepatitis syndrome. Southern Med J, 37: 251-7, 1944.

Carbone KM. Borna disease virus and human disease. Clin Microbiol Rev, 14: 513-27, 2001.

Chandra PS, Desai G, Ranjan S. HIV \& psychiatric disorders. Indian J Med Res, 121: 451-67, 2005.

Chuang HT, Jason GW, Pajurkova EM, Gill MJ. Psychiatric morbidity in patients with HIV infection. Can J Psychiatry, 37: 109-15, 1992.

Ciesla JA, Roberts JE. Meta-analysis of the relationship between HIV infection and risk for depressive disorders. Am J Psychiatry, 158: 725-30, 2001

Cooke RG, Warsh JJ, Hasey GM, McLaughlin BJM, Jorna T. EpsteinBarr virus antibodies and severity of depression. Biol Psychiatry, 29: 621-3, 1991.

DuBois RE, Seeley JK, Brus I, Sakamoto K, Ballow M, Harada S, et al. Chronic mononucleosis syndrome. Southern Med J, 77 1376-7, 1984.

Dwight MM, Kowdley KV, Russo JE, Ciechanowski PS, Larson AM, Katon WJ. Depression, fatigue, and functional disability in patients with chronic hepatitis C. J Psychosom Res, 49: 311-7, 2000

El-Serag HB, Kunik M, Richardson P, Rabeneck L. Psychiatric disorders among veterans with hepatitis C infection. Gastroenterol, 123 476-82, 2002.

Evans DL, Have TRT, Douglas SD, Gettes DR, Morrison M, Chiappini MS, et al. Association of depression with viral load, CD8 T lymphocytes, and natural killer cells in women with HIV infection. Am J Psychiatry, 159: 1752-9, 2002.

Fireman M, Indest DW, Blackwell A, Whitehead AJ, Hauser P. Addressing tri-morbidity (hepatitis $\mathrm{C}$, psychiatric disorders, and substance use): the importance of routine mental health screening as a component of a co-management model of care. Clin Infect Dis, 40 suppl 5: S286-91, 2005.
Flewett TH. Postinfluenzal depression. Br Med J, 2: 817, 1976.

Fukunishi I, Matsumoto T, Negishi M, Hayashi M, Hosaka T, Moriya H. Somatic complaints associated with depressive symptoms in HIVpositive patients. Psychother Psychosom, 66: 248-51, 1997.

Fulk LJ, Kane BE, Phillips KD, Bopp CM, Hand GA. Depression in HIV-infected patients: allopathic, complementary, and alternative treatments. J Psychosom Res, 57: 339-51, 2004.

Goggin K, Engelson ES, Rabkin JG, Kotler DP. The relationship of mood, endocrine, and sexual disorders in human immunodeficiency virus positive (HIV+) women: an exploratory study. Psychosom Med, 60: 11-6, 1998

Goulding C, O'Connell P, Murray FE. Prevalence of fibromyalgia, anxiety and depression in chronic hepatitis $C$ virus infection: relationship to RT-PCR status and mode of acquisition. Eur $J$ Gastroenterol Hepatol, 13: 507-11, 2001.

Halonen PE, Rimon R, Arohonka K, Jäntti V. Antibody levels to herpes simplex type I, measles and rubella viruses in psychiatric patients. Br J Psychiatry, 125: 461-5, 1974.

Hashimoto F, Kellner R, Kapsner CO. Upper respiratory tract infections increase self-rated hostility and distress. Int J Psychiatry Med, 17: 41-7, 1987.

Hendler N, Leahy W. Psychiatric and neurologic sequelae of infectious mononucleosis. Am J Psychiatry, 135: 842-4, 1978.

Herbert TB, Cohen S. Depression and immunity: a meta-analytic review. Psychol Bull, 113: 472-86, 1993

Hotopf MH, Wessely S. Viruses, neurosis and fatigue. J Psychosom Res, 38: 499-514, 1994.

Ickovics JR, Hamburger ME, Vlahov D, Schoenbaum EE, Schuman $\mathrm{P}$, Boland RJ, et al. Mortality, CD4 cell count decline, and depressive symptoms among HIV-seropositive women: longitudinal analysis from the HIV epidemiology research study. JAMA, 285: 1466-74, 2001.

Influenza and the nervous system [editorial]. Br Med J (5745): 357-8, 1971 Isaacs R. Chronic infectious mononucleosis. Blood, 3: 858-61, 1948.

James ME. HIV seropositivity diagnosed during pregnancy: psychosocial characterization of patients and their adaptation. Gen Hosp Psychiatry, 10: 309-16, 1988.

Johnson ME, Fisher DG, Fenaughty A, Theno SA. Hepatitis $C$ virus and depression in drug users. Am J Gastroenterol, 93: 785-9, 1998.

Katon W, Schulberg H. Epidemiology of depression in primary care. Gen Hosp Psychiatry, 14: 237-47, 1992.

Katon W, Russo J, Ashley RL, Buchwald D. Infectious mononucleosis: psychological symptoms during acute and subacute phases of illness. Gen Hosp Psychiatry, 21: 21-9, 1999.

Kelly B, Raphael B, Judd F, Perdices M, Kernutt G, Burrows GD, et al. Psychiatric disorder in HIV infection. Aust N Z J Psychiatry, 32: 441-53, 1998

Kim YK, Kim SH, Choi S, Ko Y, Kim L, Lee MS, et al. Failure to demonstrate Borna disease virus genome in peripheral blood mononuclear cells from psychiatric patients in Korea. J Neurovirol, 5: 196-9, 1999.

Koskinas J, Merkouraki P, Manesis E, Hadziyannis S. Assessment of depression in patients with chronic hepatitis: effect of interferon treatment. Dig Dis, 20: 284-8, 2002.

Lambore S, McSherry J, Kraus AS. Acute and chronic symptoms of mononucleosis. J Fam Pract, 33: 33-7, 1991.

Lee DH, Jamal H, Regestein FG, Perrillo RP. Morbidity of chronic hepatitis $C$ as seen in a tertiary care medical center. Dig Dis Sci, 42: 186-91, 1997.

Lehman CL, Cheung RC. Depression, anxiety, post-traumatic stress, and alcohol-related problems among veterans with chronic hepatitis $C$. Am J Gastroenterol, 97: 2640-6, 2002. 
Leserman J, Jackson ED, Petitto JM, Golden RN, Silva SG, Perkins D, et al. Progression to AIDS: the effects of stress, depressive symptoms, and social support. Psychosom Med, 61: 397-406, 1999.

Lipsitz JD, Williams JBW, Rabkin JG, Remien RH, Bradbury M, El Sadr W, et al. Psychopathology in male and female intravenous drug users with and without HIV infection. Am J Psychiatry, 151: 1662-8, 1994

Lovisi GM, Morgado AF. Suporte social e distúrbios psiquiátricos em mulheres infectadas pelo HIV. J Bras Psiq, 45: 593-9, 1996.

Low spirits after virus infections [editorial]. Br Med J, 2: 440, 1976.

Lycke E, Norrby R, Björn-Erik R. A serological study on mentally ill patients. Br J Psychiatry, 124: 273-9, 1974.

Lyketsos CG, Hoover DR, Guccione M, Dew MA, Wesch JE, Bing $E G$, et al. Depressive symptoms over the course of HIV infection before AIDS. Soc Psychiatry Psychiatr Epidemiol, 31: 212-9, 1996a.

Lyketsos CG, Hoover DR, Guccione M, Dew MA, Wesch JE, Bing EG, et al. Changes in depressive symptoms as AIDS develops. Am J Psychiatry, 153: 1430-7, 1996b.

Maj M, Janssen R, Starace F, Zaudig M, Satz P, Sughondhabirom B, et al. WHO neuropsychiatric AIDS study, cross-sectional phase I. Arch Gen Psychiatry, 51: 39-49, 1994.

Malbergier A, Andrade AG. Transtornos depressivos em usuários de drogas injetáveis infectados pelo HIV: um estudo controlado. Rev Bras Psiquiatr, 21: 217-24, 1999.

Malbergier A, Schöeffel AC. Tratamento de depressão em indivíduos infectados pelo HIV. Rev Bras Psiquiatr, 23: 160-7, 2001.

Martini GA, Strohmeyer G. Posthepatitis syndromes. Clin Gastroenterol, 3: 377-90, 1974.

McDaniel JS, Fowlie E, Summerville MB, Farber EW, Cohen-Cole SA. An assessment of rates of psychiatric morbidity and functioning in HIV disease. Gen Hosp Psychiatry, 17: 346-52, 1995.

Meijer A, Zakay-Rones Z, Morag A. Post-influenzal psychiatric disorder in adolescents. Acta Psychiatr Scand, 78: 176-81, 1988.

Menninger KA. Influenza and melancholy. J Nerv Ment Dis, 53: 257-81, 1921.

Morrison MF, Petitto JM, Have TT, Gettes DR, Chiappini MS, Weber AL, et al. Depressive and anxiety disorders in women with HIV infection. Am J Psychiatry, 159: 789-96, 2002.

Muir AJ, Provenzale D. A descriptive evaluation of eligibility for therapy among veterans with chronic hepatitis C virus infection. J Clin Gastroenterol, 34: 268-71, 2002.

Pariante CM, Orrú MG, Baita A, Farci MG, Carpiniello B. Treatment with interferon- $\alpha$ in patients with chronic hepatitis and mood or anxiety disorders. Lancet, 354: 131-2, 1999.

Penzak SR, Reddy YS, Grimsley SR. Depression in patients with HIV infection. Am J Health-System Pharmacy, 57: 376-89, 2000.

Pergami A, Gala C, Burgess A, Durbano F, Zanello D, Riccio M, et al. The psychosocial impact of HIV infection in women. J Psychosom Res, 37: 687-96, 1993.

Perkins DO, Stern RA, Golden RN, Murphy C, Naftolowitz D, Evans DL. Mood disorders in HIV infection: prevalence and risk factors in a nonepicenter of the AIDS epidemic. Am J Psychiatry, 151: 233-6, 1994.

Pokorny AD, Rawls WE, Adam E, Mefferd RB. Depression, psychopathy, and herpesvirus type I antibodies. Arch Gen Psychiatry, 29: 820-2, 1973.

Proietti ABCF. HTLV I/II. 3 ed. Belo Horizonte: Fundação Centro de Hematologia e Hemoterapia de Minas Gerais, 2000.
Rabkin JG, Ferrando SJ, Jacobsberg LB, Fishman B. Prevalence of axis I disorders in an AIDS cohort: a cross-sectional, controlled study. Compr Psychiatry, 38: 146-54, 1997a.

Rabkin JG, Goetz RR, Remien RH, Williams JBW, Todak GMSW, Gorman JM. Stability of mood despite HIV illness progression in a group of homosexual men. Am J Psychiatry, 154: 231-8, $1997 \mathrm{~b}$.

Rimon R, Halonen P. Herpes simplex virus infection and depressive illness. Dis Nerv System, 30: 338-40, 1969.

Rosenberger PH, Bornstein RA, Nasrallah HA, Para MF, Whitaker $C C$, Fass RJ, et al. Psychopathology in human immunodeficiency virus infection: lifetime and current assessment. Compr Psychiatry, 34: 150-8, 1993.

Sherlock S, Walshe V. The post-hepatitis syndrome. Lancet, ii: 482-4, 1946

Sherman M, Driver HS, Devins G, Shapiro C. Assessment of quality of life in patients with chronic hepatitis $C$ using a range of subjective fatigue assessment tools. Hepatol, 30: 204A, 1999.

Sinanan K, Hillary I. Post-influenzal depression. Br J Psychiat, 138: 131-3, 1981.

Singh N, Gayowski T, Wagener MM, Marino IR. Vulnerability to psychologic distress and depression in patients with end-stage liver disease due to hepatitis $C$ virus. Clin Transplantation, 11: 406-11, 1997.

Singh N, Gayowski T, Wagener MM, Marino IR. Quality of life, functional status, and depression in male liver transplant recipients with recurrent viral hepatitis $C$. Transplantation, 67 : 69-72, 1999.

Stumpf BP, Catalan-Soares B, Carneiro-Proietti AB, Namen-Lopes S, Proietti FA, Rocha FL. Depression in HTLV-1 infected individuals: initial reports from the GIPH cohort in Belo Horizonte, Brazil. AIDS Research and Human Retroviruses 21: 465, 2005.

Taruschio G, Santarini F, Sica G, Dragoni C, Migliorini S, Andreone $C C$, et al. Psychiatric disorders in hepatitis $C$ virus related chronic liver disease. Gastroenterol, 110: A1342, 1996.

Tostes MA, Chalub M, Botega NJ. The quality of life of HIV-infected women is associated with psychiatric morbidity. AIDS Care, 16: 177-86, 2004.

Turrina C, Fiorazzo A, Turano A, Cacciani P, Regini C, Castelli F, et al. Depressive disorders and personality variables in HIV positive and negative intravenous drug-users. J Affect Disord, 65: 45-53, 2001.

Weisse CS. Depression and immunocompetence: a review of the literature. Psychol Bull, 111: 475-89, 1992.

Wessely S, Pariante C. Fatigue, depression and chronic hepatitis C infection. Psychol Med, 32: 1-10, 2002.

White PD, Lewis SW. Delusional depression after infectious mononucleosis. Br Med J, 295: 97-8, 1987.

White PD, Thomas JM, Amess J, Crawford DH, Grover SA, Kangro $\mathrm{HO}$, et al. Incidence, risk and prognosis of acute and chronic fatigue syndromes and psychiatric disorders after glandular fever. Br J Psychiatry, 173: 475-81, 1998.

White PD. Do viruses cause depressive illness? Gen Hosp Psychiatry, 21: 18-20, 1999

Williams JBW, Rabkin JG, Remien RH, Gorman JM, Ehrhardt AA. Multidisciplinary baseline assessment of homosexual men with and without human immunodeficiency virus infection. Arch Gen Psychiatry, 48: 124-30, 1991.

Zdilar D, Franco-Bronson K, Buchler N, Locala JA, Younossi ZM. Hepatitis C, interferon alfa, and depression. Hepatol, 31: 1207-11, 2000. 